\title{
DESARROLLO DE HIPEREMIA CONJUNTIVAL TRAS EL EMPLEO DE LA COMBINACIÓN FIJA DE LATANOPROST/TIMOLOL: REVISIÓN SISTEMÁTICA Y METAANÁLISIS DE ENSAYOS CLÍNICOS PUBLICADOS
}

\author{
CONJUNTIVAL HYPEREMIA WITH THE USE OF A FIXED \\ COMBINATION OF LATANOPROST/TIMOLOL: SYSTEMATIC \\ REVIEW AND META-ANALYSIS OF CLINICAL TRIALS
}

\author{
VINUESA-SILVA JM ${ }^{1}$, VINUESA-SILVA I ${ }^{2}$, PINAZO-DURÁN MD ${ }^{3}$, SOTO-ÁLVAREZ J ${ }^{4}$, \\ DELGADO-ORTEGA L ${ }^{5}$, DÍAZ-CEREZO $\mathrm{S}^{5}$
}

\section{RESUMEN}

Objetivo: Valorar la asociación de hiperemia conjuntival con el uso de la combinación fija de latanoprost/timolol en el tratamiento del glaucoma, a través de una revisión sistemática y de un metaanálisis. Métodos: Se efectuó una búsqueda de los ensayos clínicos publicados de latanoprost/timolol y distintos comparadores en las bases de datos Medline, Embasse y Cochrane Controlled Clinical Trials Register, entre 2000 y 2007. La medida para valorar el tamaño del efecto ha sido la odds ratio (OR) y su intervalo de confianza (IC) del $95 \%$, habiéndose calculado mediante el modelo de efectos fijos de Mantel-Haenszel y el de efectos aleatorios de Der Simonian and Laird. Para valorar la existencia de heterogeneidad entre los estudios, se llevó a cabo la prueba Q de Cochran así como el cálculo del índice $I^{2}$. Los porcentajes de hiperemia hallados se han comparado a través de la prueba de Chi-cuadrado.

\begin{abstract}
Purpose: To asses the association of conjuntival hyperemia with the use of a fixed combination of latanoprost/timolol, through a systematic review and metaanalysis of clinical trials in patients with glaucoma.

Methods: A systematic review of published clinical trials of latanoprost/timolol and other competitors was conducted in Medline, Embasse and Cochrane Controlled Clinical Trials Register, between 2000 and 2007. Statistical analysis included calculation of the odds ratio (OR) with its $95 \%$ confidence interval (CI) using the fixed effects model of Mantel-Haenszel and the random effects model of Der Simonian and Laird. To assess the heterogeneity between trials the Cochran Q test and the $I^{2}$ rate were calculated. The conjuntival hyperemia rates obtained were compared with the Chi-square test.

Results: A total of 8 clinical trials comparing latanoprost/timolol fixed combination with different
\end{abstract}

\footnotetext{
Recibido: 15/10/08. Aceptado: 1/4/09.

1 Doctor en Medicina. Facultad de Medicina. Universidad de Salamanca. Salamanca. España.

2 Doctor en Medicina. Servicio de Oftalmología. Hospital Punta de Europa. Algeciras. España.

3 Doctor en Medicina. Unidad Investigación Oftalmológica Santiago Grisolía. Valencia. España.

4 Doctor en Medicina. Departamento de Investigación de Resultados en Salud, Unidad Médica, Pfizer España.

5 Licenciado en Farmacia. Departamento de Investigación de Resultados en Salud, Unidad Médica, Pfizer España.

Correspondencia:

Silvia Díaz Cerezo

Departamento de Investigación de Resultados en Salud

Unidad Médica, Pfizer España

Avda. de Europa, 20-B

Parque Empresarial La Moraleja, Alcobendas

28018 Madrid

España

E-mail: silvia.diaz@pfizer.com
} 
Resultados: Se encontraron 8 ensayos clínicos que comparaban latanoprost/timolol con distintas opciones terapéuticas. La heterogeneidad de los estudios fue moderada (Q: 14,64; df=7; $\mathrm{p}=0,041$; $\left.\mathrm{I}^{2}=52,2 \%\right)$ por lo que se utilizó el modelo de efectos aleatorios. La OR final fue de 0,47 (IC 95\%: $0,24-0,90) ; p=0,024$. La incidencia total de hiperemia fue de $2,9 \%$ en el grupo latanoprost/timolol y de $7,0 \%$ para los comparadores $(\mathrm{p}<0,0001)$.

Conclusiones: El uso de latanoprost/timolol se asocia con una disminución significativa de la aparición de hiperemia conjuntival del 53\% (IC 95\%: $10 \%-76 \%$ ) frente a otras opciones de tratamiento en el manejo del glaucoma.

Palabras clave: Hiperemia conjuntival, glaucoma, latanoprost, timolol, metaanálisis. therapeutic options were found. As trial heterogeneity was moderate $\left(\mathrm{Q}: 14.64 ; \mathrm{df}=7 ; \mathrm{p}=0.041 ; I^{2}=\right.$ $52.2 \%)$ a random effects model was used. The final OR was 0.47 (CI 95\%: 0.24-0.90); $\mathrm{p}=0.024$. The total conjuntival hyperemia incidence was $2.9 \%$ in the latanoprost/timolol group and $7.0 \%$ for the competitors $(p<0.0001)$.

Conclusions: The use of a fixed combination of latanoprost/timolol is associated with a significant reduction (53\%; CI 95\%: 10\%-76\%) in the development of conjuntival hyperemia against the other compared options for the treatment of glaucoma (Arch Soc Esp Oftalmol 2009; 84: 199-208).

Key words: Conjuntival hyperemia, glaucoma, latanoprost, timolol, meta-analysis.

\section{INTRODUCCIÓN}

Según la Organización Mundial de la Salud (OMS), el glaucoma es la 2. ${ }^{a}$ causa de ceguera en los países desarrollados afectando a 60 millones de personas y se cree que el $50 \%$ de los casos de glaucoma están aún sin diagnosticar (1). Las cifras de incidencia aumentan proporcionalmente con la edad (2-4) y se estima que la prevalencia en la población general es de alrededor del 2\%. Si nos centramos en la población mayor de 50 años esta cifra aumenta al 3\%, siendo del 23\% de los enfermos mayores de 75 años.

El diagnóstico precoz es fundamental para evitar la progresión de la enfermedad, pero suele ser complicado por tratarse de una enfermedad inicialmente indolora y sin síntomas. El objetivo ideal sería identificar a los individuos en la fase inicial de la enfermedad y a aquéllos susceptibles de padecerla que presenten factores de riesgo, como historia familiar, miopía elevada, diabetes, hipertensión arterial, edad y aumento de la presión intraocular (PIO) (5).

Los tratamientos farmacológicos se basan en la disminución de la PIO para retrasar o prevenir la progresión a glaucoma en pacientes con hipertensión ocular o para ralentizar su progresión en el caso de aquellos que ya presenten la enfermedad (6). El tratamiento farmacológico de elección es por vía tópica y, al tratarse de una enfermedad crónica, debe seguirse de por vida, junto con revisiones periódicas de la PIO y del campo visual $(7,8)$. Por ello, a la hora de elegir un tratamiento, es fundamental tener en cuenta, además de la eficacia y seguridad del fármaco, que el paciente sea capaz de cumplir el tratamiento de forma prolongada, ya que en aquellos pacientes que interrumpen el tratamiento se ha observado un aumento de la PIO, que puede conllevar una progresión de la enfermedad hacia la ceguera (9).

Actualmente, la Sociedad Europea del Glaucoma (EGS) recomienda que la primera línea de tratamiento consista en la monoterapia con un agente hipotensor tópico y que su elección dependa no sólo del perfil de eficacia y seguridad del medicamento sino también de la calidad de vida del paciente, del coste y del cumplimiento. Si un fármaco no alcanza el nivel de PIO buscado debe sustituirse por otro, y si la monoterapia no consigue el nivel deseado de ésta, deben asociarse varios fármacos $(7,8)$.

El cumplimiento del tratamiento por parte del paciente depende de muchos factores (satisfacción con el tratamiento, coste, fácil dosificación, conocimiento sobre su enfermedad y de las consecuencias de interrumpir el tratamiento) pero principalmente de los efectos secundarios locales y sistémicos que le provoque (10). Si comparamos los beta-bloqueantes con las prostaglandinas, los primeros manifiestan pocos efectos locales, pero pueden provocar efectos sistémicos importantes como el agravamiento del asma bronquial, de la enfermedad pulmonar obstructiva crónica, la insuficiencia cardíaca 
y de la arritmia bradicárdica entre otros. En el caso de las prostaglandinas ocurre lo contrario; apenas presentan efectos sistémicos, pero entre los locales se puede manifestar hiperemia conjuntival, pigmentación iridiana, engrosamiento y aumento de las pestañas, queratitis punteada, uveítis anterior, edema macular cistoide (11).

La hiperemia conjuntival es el efecto secundario más común de las prostaglandinas (12) y debe ser considerado por los oftalmólogos porque puede desencadenar el abandono del tratamiento (13). Dada la importancia de este efecto adverso y dado que la asociación de una prostaglandina con timolol es uno de los tratamientos de 2. ${ }^{a}$ línea más empleados, el objetivo de este trabajo fue llevar a cabo una revisión sistemática y metaanálisis de los ensayos clínicos publicados hasta el momento, comparando los efectos de hiperemia que produce la combinación fija de latanoprost/timolol frente a otros posibles tratamientos (latanaprost y timolol por separado y otros medicamentos hipotensores) en pacientes con hipertensión ocular o glaucoma.

\section{SUJETOS, MATERIAL Y MÉTODOS}

\section{Estrategia de búsqueda}

Los artículos fueron identificados a través de la búsqueda de los ensayos clínicos publicados en las bases de datos Medline, Embase y Cochrane Controlled Clinical Trials Register, entre 2000 y 2007, en los que se hubieran comparado la combinación fija de latanoprost/timolol frente a otras opciones terapéuticas para el tratamiento del glaucoma o la hipertensión intraocular.

En la búsqueda se usaron las siguientes palabras claves: glaucoma, hipertensión intraocular, aleatorización, ensayo clínico, latanoprost/timolol e hiperemia conjuntival.

\section{Selección de estudios}

Dos investigadores independientes realizaron la búsqueda de los trabajos publicados y seleccionaron los artículos relevantes acordes a los criterios fijados para la inclusión de los estudios publicados en la revisión sistemática: a) ensayos clínicos comparativos (excluyendo diseños cruzados); b) existencia de un control activo en el estudio; c) valora- ción de la incidencia de hiperemia conjuntival debida a los tratamientos administrados.

Para evaluar los artículos encontrados, inicialmente se revisó el título y el resumen de los mismos para ver si cumplían o no con los criterios de inclusión. En aquéllos que en principio cumplían con estos criterios se revisó el artículo completo para estar seguro que cumplían fielmente con los criterios de inclusión y que, por lo tanto, tenían que ser incluidos en la revisión sistemática.

\section{Extracción de datos}

Los dos investigadores llevaron a cabo la extracción de los datos, rellenando una hoja de codificación creada ad hoc para el estudio. Adicionalmente, evaluaron la calidad metodológica de los ensayos que fueron incluidos, como se describe más tarde. Todas las diferencias encontradas fueron discutidas y solucionadas por consenso entre los dos investigadores, y en los casos en que no pudieron llegar a una solución consensuada se pidió la intervención de un tercer investigador.

Se diseñó una tabla para resumir de forma clara la información obtenida de cada uno de los artículos seleccionados, en la que se indican los autores del ensayo, el tratamiento evaluado, el tratamiento comparador, el número de pacientes en los que se evaluó el grado de hiperemia, su edad media y el porcentaje de hiperemia presentado en cada grupo de pacientes.

\section{Evaluación de la calidad del estudio}

Los investigadores determinaron la calidad de los estudios utilizando la escala Jadad (14). Se trata de un instrumento validado, en la que los estudios son puntuados entre 0 y 8 puntos según sus características: aleatorización (1 ó 2 puntos dependiendo de si se describe el método usado), enmascaramiento (1 ó 2 puntos dependiendo de si se describe el método usado), criterios de inclusión y de exclusión descritos (1 punto), abandonos o retiradas especificados (1 punto) y método de evaluación de efectos adversos descrito (1 punto). El resultado final nos da una estimación de la validez de los ensayos clínicos utilizados para el desarrollo del metaanálisis; cuanto más alto, más válido y fiable es el ensayo. 


\section{Análisis estadístico}

Todo el análisis estadístico de los datos incluidos en el meta-análisis se realizó con el programa «Comprehensive Meta-Analysis» versión 2.2 (Biostat, Inc., Englewood, New Yersey; www.metaanalysis.com).

La heterogeneidad entre los ensayos se analizó mediante la prueba Q de Cochran y a través del cálculo del índice $I^{2}$, el cual permite conocer cómo la posible heterogeneidad existente puede afectar a las conclusiones del metaanálisis (15). Los valores de $I^{2}$ del $50 \%$ o más, indican un nivel considerable de heterogeneidad. Para la prueba Q de Cochran se situó la significación estadística en $p<0,1$ en vez de $\mathrm{p}<0,05$, teniendo en cuenta su baja potencia de contraste cuando el número de estudios incluido es bajo.

Para valorar el tamaño del efecto se utilizó la odds ratio (OR). Las OR de los diferentes ensayos clínicos se combinaron utilizando el modelo de efectos fijos de Mantel-Haenszel (para ensayos clínicos homogéneos) (16) y el modelo de efectos aleatorios de Der Simonian and Laird (para ensayos clínicos heterogéneos) (17). Los resultados obtenidos se representaron en un gráfico tipo forest plot. En este gráfico se presentan los valores de la OR con su correspondiente intervalo de confianza del 95\% para cada estudio individual y el valor global o agregado para el metaanálisis, estableciéndose una significación estadística a partir de p<0,05.

El posible sesgo de publicación, se evaluó mediante la inspección visual del funnel plot o gráfico de embudo. Este gráfico va a ordenar los resultados a partir de la precisión de su medida, y podremos comprobar si existen estudios con poca precisión y resultados extremos, tanto a favor del grupo de latanoprost/timolol como en contra. Si sólo observamos resultados extremos de estudios con poca precisión (normalmente con escasa muestra de sujetos) a favor de latanoprost/timolol, pero no a favor del grupo comparador, podríamos suponer que éstos (los estudios con resultado negativo) existen pero no han sido publicados. Cuando las muestras son pequeñas el error aleatorio del estudio es alto y por tanto, los resultados deberían ir tanto a favor como en contra (debido al efecto del azar). Cuando no existe sesgo de publicación los puntos del gráfico tienden a distribuirse en forma de un embudo invertido. Si por el contrario, existe este sesgo, la nube de puntos aparecerá deformada en uno de sus extremos (18).
Con el fin de analizar la robustez de los resultados y, para comprobar si alguno de los estudios está ejerciendo una influencia decisiva sobre los mismos, se realizó un análisis de sensibilidad extrayendo sistemáticamente y por separado cada uno de los ensayos clínicos incluidos en el metaanálisis. Si los resultados así obtenidos son similares, tanto en dirección como en magnitud del efecto y significación estadística, podemos concluir que el análisis es robusto.

Se ha seguido la guía de QUÓROM (Quality of Reporting of Meta-analysis) para presentar y discutir los resultados del metaanálisis (19).

Adicionalmente se calculó la frecuencia de aparición de hipermia en cada grupo (latanprost/timolol y resto de comparadores) y se calculó si existían diferencias estadísticamente significativas mediante la prueba de Chi Cuadrado de Mantel Haenszel. El grado de intensidad de la posible asociación entre la aparición de la hipermia y el tratamiento utilizado se calculó mediante la estimación del riesgo relativo (RR) ponderado por el método de Mantel Haenszel (el más correcto en el caso de estimaciones con pocos sujetos, como ocurre en nuestro estudio) (20). Estos cálculos se llevaron a cabo con el programa de análisis estadístico SPSS v.14.

\section{RESULTADOS}

\section{Búsqueda literaria}

Se identificaron un total de 19 artículos, potenciales candidatos a ser incluidos en el metaanálisis, en los que se habían evaluado las asociaciones latanoprost/timolol frente otras opciones terapéuticas. De todos ellos, por los criterios de exclusión anteriormente mencionados, un total de 11 artículos fueron excluidos del metaanálisis; 2 porque no eran ensayos clínicos $(21,22), 1$ porque no tenía control activo (23), 5 por ser ensayos clínicos de diseño cruzado (24-28) y los 3 restantes porque no se notificaba la incidencia de hiperemia (29-31). La concordancia entre los dos investigadores en cuanto a la inclusión de los estudios en el análisis fue buena (Indice Kappa $=0,8$ ). Las discrepancias fueron resueltas por el tercer investigador.

Finalmente, quedaron 8 ensayos clínicos que fueron incluidos en el metaanálisis; todos incluyeron pacientes con hipertensión ocular o glaucoma y aportaban datos específicos de hiperemia de un 
total de 2600 pacientes (32-39). El flujo del análisis de los ensayos clínicos excluidos e incluidos se muestra en la figura 1.

\section{Características de los ensayos}

La tabla I muestra las características de los ensayos clínicos incluidos en el metaanálisis.

$\mathrm{Al}$ comienzo de los ensayos se contaba con una población aleatorizada de 2.511 pacientes que fueron evaluados por un periodo medio de 4,72 meses (min.=21 días, máx.= 12 meses). 1.761 pacientes fueron diagnosticados de glaucoma de ángulo abierto, 549 tuvieron hipertensión intraocular y 201 algún otro tipo de glaucoma. Durante el transcurso de los ensayos clínicos, el total de pacientes de los que se obtuvieron datos de hiperemia conjuntival y que fueron considerados en este metaanálisis fue de 2600 pacientes. De ellos, un total de 1156 fueron tratados con la combinación fija de latanoprost/timolol y el resto fueron tratados con



Fig. 1: Diagrama de flujo de los ensayos clínicos incluidos y excluidos en el metaanálisis. los diferentes comparadores: 460 sólo con latanoprost, 289 sólo con timolol, 50 sólo con travoprost, 162 con la combinación no fija de brimonidina y timolol, 254 con la combinación no fija de latanoprost y timolol, 22 con la combinación no fija de travoprost y brinzolamida y 207 con la combinación fija de travoprost y timolol.

La frecuencia de aparición de hiperemia ponderada por el número de sujetos de cada estudio fue del 2,9\% en el grupo de latanoprost/timolol y del $7,0 \%$ en el grupo de comparadores; $p<0,0001$.

Dado que se encontraron diferencias estadísticamente significativas se calculó el RR ponderado por el método de Mantel Haenszel: RR = 0,44 (IC 95\%: 0,30-0,63); $\mathrm{p}=0,00001$. Por lo tanto, la utilización de latanoprost/timolol reduce el riesgo de hipermia en un $56 \%$ respecto a la utilización del resto de comparadores analizados en nuestro estudio.

\section{Resultados del metaanálisis}

La prueba Q de Cochran indicó que existía cierto grado de heterogeneidad entre los estudios $(\mathrm{Q}=$ $14,64 ; \mathrm{p}=0,041)$. Este hallazgo fue corroborado por el valor del índice de $I^{2}=52,2 \%$, que indica la existencia de una heterogeneidad moderada. Dados estos valores, el cálculo de la OR final se llevó a cabo mediante el modelo de efectos aleatorios, que es más conservador y tiene en cuenta esta heterogeneidad. Los resultados de este último modelo, señalan que el uso de la combinación fija de latanoprost/timolol frente a otros comparadores se asoció con un menor porcentaje de hiperemia conjuntival $(\mathrm{OR}=0,467 ; 95 \%$ CI: 0,242 - 0,905, $\mathrm{p}=0,024)$. Las OR de cada uno de los estudios y la OR final combinada calculada con ambos modelos estadísticos (de efectos fijos y aleatorios) se presentan en la figura 2 .

\section{Análisis de sensibilidad}

Los análisis de sensibilidad realizados mostraron que la OR media agregada podía oscilar entre 0,39 y 0,50 dependiendo del ensayo clínico excluido del metaanálisis. Ninguno de ellos tuvo un impacto significativo en la estimación global de la OR ya que en todos los casos el grupo de latanoprost/timolol presentó un grado de hipermia significativamente menor vs. el grupo comparador (tabla II). 
Tabla I. Principales características de los ensayos clínicos incluidos en el metaanálisis

\begin{tabular}{|c|c|c|c|c|c|c|c|c|c|}
\hline \multirow[t]{2}{*}{ Estudio } & \multirow{2}{*}{$\begin{array}{c}\text { Tratamiento } \\
\text { Evaluado }\end{array}$} & \multirow{2}{*}{$\begin{array}{l}\text { Grupo } \\
\text { control }\end{array}$} & \multirow[t]{2}{*}{ N. ${ }^{\circ}$ Pacientes } & \multicolumn{3}{|c|}{ Pacientes con hiperemia } & \multirow[t]{2}{*}{ Duración* } & \multirow{2}{*}{$\begin{array}{r}\text { Edad Media } \\
\text { Ponderada }\end{array}$} & \multirow{2}{*}{$\begin{array}{l}\text { Puntuación } \\
\text { Jadad }\end{array}$} \\
\hline & & & & LT/TM & $\mathrm{TR} / \mathrm{TM}$ & Control & & & \\
\hline Pfeiffer N (30) & LT/TM & LT ; TM & $\begin{array}{l}\mathrm{LT} / \mathrm{TM}=140 \\
\mathrm{LT}=147 ; \mathrm{TM}=149\end{array}$ & $2,86 \%$ & & $1,01 \%$ & $6 \mathrm{~m}$ & 64 & 7 \\
\hline $\begin{array}{l}\text { Higginbotham EJ, } \\
\text { et al (31) }\end{array}$ & $\mathrm{LT} / \mathrm{TM}$ & $\mathrm{LT} ; \mathrm{TM}$ & $\begin{array}{l}\mathrm{LT} / \mathrm{TM}=138 \\
\mathrm{LT}=140 ; \mathrm{TM}=140\end{array}$ & $7,97 \%$ & & $10,71 \%$ & $6 \mathrm{~m}$ & 62 & 8 \\
\hline $\begin{array}{l}\text { Olander K, } \\
\text { et al (32) }\end{array}$ & $\mathrm{LT} / \mathrm{TM}$ & LT & $\begin{array}{l}\mathrm{LT} / \mathrm{TM}=175 \\
\mathrm{LT}=173\end{array}$ & $0,6 \%$ & & $1,2 \%$ & $21 \mathrm{~d}$ & 64 & 8 \\
\hline $\begin{array}{l}\text { Franks WA, } \\
\text { et al (33) }\end{array}$ & LT/TM & $\mathrm{TR}$ & $\begin{array}{l}\mathrm{LT} / \mathrm{TM}=56 \\
\mathrm{TR}=50\end{array}$ & $1,8 \%$ & & $9,3 \%$ & $6 \mathrm{~s}$ & $\begin{array}{l}N=37 ;<65 \\
N=69 ; \geq 65\end{array}$ & 6 \\
\hline $\begin{array}{l}\text { Garcia-Sánchez J, } \\
\text { et al (34) }\end{array}$ & LT/TM & BR y TM & $\begin{array}{l}\mathrm{LT} / \mathrm{TM}=163 \\
\mathrm{BR} \text { y } \mathrm{TM}=162\end{array}$ & $0,61 \%$ & & $2,47 \%$ & $6 \mathrm{~m}$ & 65 & 5 \\
\hline $\begin{array}{l}\text { Diestelhorst M, } \\
\text { et al (35) }\end{array}$ & LT/TM & LT y TM & $\begin{array}{l}\mathrm{LT} / \mathrm{TM}=262 \\
\mathrm{LT} \text { y } \mathrm{TM}=254\end{array}$ & $3,1 \%$ & & $8,7 \%$ & $12 \mathrm{~s}$ & 65 & 8 \\
\hline $\begin{array}{l}\text { Martínez de la Casa JM, } \\
\text { et al (36) }\end{array}$ & LT/TM & TR y BZ & $\begin{array}{l}\mathrm{LT} / \mathrm{TM}=22 \\
\mathrm{TR} \text { y } \mathrm{BZ}=22\end{array}$ & $13,6 \%$ & & $18,2 \%$ & $3 \mathrm{~m}$ & 63 & 5 \\
\hline $\begin{array}{l}\text { Topouzis F, } \\
\text { et al (37) }\end{array}$ & LT/TM & TR/TM & $\begin{array}{l}\mathrm{LT} / \mathrm{TM}=200 \\
\mathrm{TR} / \mathrm{TM}=207\end{array}$ & $2,5 \%$ & $15,0 \%$ & & $12 \mathrm{~m}$ & 65 & 8 \\
\hline
\end{tabular}

Dosis utilizadas en los ensayos clínicos: monoterapias (Latanaprost $(\mathrm{LT})=0,005 \%$, Timolol $(\mathrm{TM})=0,5 \%$, Travoprost $(\mathrm{TR})=0,004 \%$, Brimonidina $(\mathrm{BR})=0,2 \%$, Brinzolamida $(\mathrm{BZ}=0,1 \%)$ y combinaciones $(\mathrm{LT} / \mathrm{TM}=0,005 / 0,5 \%, \mathrm{TR} / \mathrm{TM}=0,004 / 0,5 \%)$.

\section{Sesgo de las publicaciones}

En base al análisis visual de gráfico de embudo, donde se ordenan los estudios a partir de la precisión de su medida, no se encontraron evidencias de sesgo (fig. 3), ya que 6 de los 8 estudios se encuentran dentro de la zona del embudo y no se aprecia un grado de asimetría importante.

\section{DISCUSIÓN}

En la actualidad, cada vez es más habitual la utilización de una combinación fija de dos fármacos en los pacientes que requieren la utilización de más de un medicamento para llegar a la PIO objetivo, lo que va a facilitar el cumplimiento del tratamiento y por lo tanto, que sea más eficaz (40). Las diferen-

\begin{tabular}{llcllc} 
Modelo & Estudio & \multicolumn{4}{c}{ Datos Estadísticos } \\
& & Odds & Límite & Límite \\
& & Ratio & Inferior & Superior & Valor p \\
& & 2,873 & 0,634 & 13,012 & 0,171 \\
& Pfeiffer N & 0,722 & 0,350 & 1,487 & 0,377 \\
& Higginbotham EJ & 0,491 & 0,044 & 5,469 & 0,563 \\
& Olander K & 0,164 & 0,018 & 1,452 & 0,104 \\
& Franks WA & 0,491 & 0,089 & 2,717 & 0,415 \\
& Garcia Sanchez J & 0,332 & 0,145 & 0,761 & 0,009 \\
& Diestelhorst M & 0,331 & 0,681 \\
& Mtnez de la Casa JM & 0,711 & 0,139 & 3,626 & 0,000 \\
Fijo & Topouzis F & 0,140 & 0,053 & 0,369 & 0,000 \\
Aleatorio & & 0,453 & 0,301 & 0,681 & 0,000 \\
& & 0,467 & 0,242 & 0,905 & 0,024
\end{tabular}

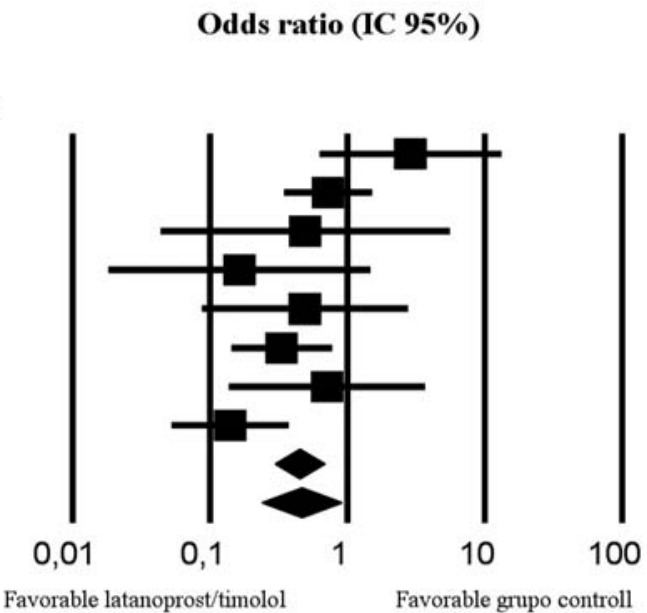

Fig. 2: La línea vertical que atraviesa el 1, representa el valor del efecto nulo. A su izquierda se presentan los estudios cuyos resultados están a favor del grupo de latanoprost/timolol y a su derecha aquella cuyos resultados están a favor del grupo comparador de otros tratamientos. 
Hiperemia asociada a latanoprost/timolol

Tabla II. Análisis de sensibilidad por exclusión sistemática de los ensayos clínicos incluidos en el metaanálisis

\begin{tabular}{|c|c|c|c|c|}
\hline Estudio excluido & Odds Ratio & Límite Inferior & Limite Superior & Valor de $\mathrm{p}$ \\
\hline Pfeiffer N & 0,391 & 0,256 & 0,598 & $<0,001$ \\
\hline Higginbotham EJ, et al & 0,363 & 0,221 & 0,597 & $<0,001$ \\
\hline Olander $\mathrm{K}$, et al & 0,452 & 0,298 & 0,684 & $<0,001$ \\
\hline Franks WA, et al & 0,470 & 0,310 & 0,712 & $<0,001$ \\
\hline Garcia-Sánchez J, et al & 0,450 & 0,296 & 0,686 & $<0,001$ \\
\hline Diestelhorst $\mathrm{M}$, et al & 0,500 & 0,312 & 0,801 & 0,004 \\
\hline Martínez de la Casa JM, et al & 0,439 & 0,288 & 0,670 & $<0,001$ \\
\hline Topouzis F, et al & 0,453 & 0,301 & 0,681 & 0,019 \\
\hline
\end{tabular}

cias que puedan existir en el cumplimiento según las distintas combinaciones fijas empleadas, podrían estar relacionadas con los efectos secundarios que provocan. En el caso de las combinaciones que incluyen una prostaglandina, el grado de hiperemia que producen puede ser, por tanto, un factor fundamental a la hora de decidir un tratamiento.

Está descrito en la literatura que de los tres análogos de prostaglandinas comercializados (latanoprost, bimatoprost y travoprost), latanoprost es la que menos hiperemia conjuntival produce, cuando se administra en monoterapias $(41,42)$.

Los resultados de este metaanálisis sugieren que el uso de la combinación fija de latanoprost/timolol se asocia con un menor grado de hiperemia que el resto de alternativas utilizadas como grupo comparador, en los distintos ensayos clínicos publicados.

Dado que en este meta-análisis solamente se incluido un ensayo clínico que compare latanoprost/timolol versus travoprost/timolol y ninguno evaluable entre latanoprost/timolol y bimatoprost/timolol, no se puede concluir que la combinación fija de latanoprost/timolol se asocie

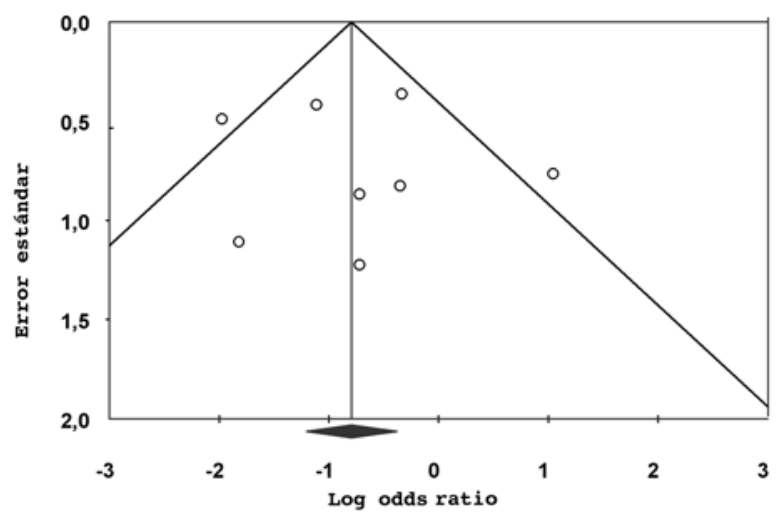

Fig. 3: Gráfico de Embudo (Funnel-Plot). con una menor aparición de hiperemia frente a las otras dos combinaciones fijas de análogos de prostaglandinas y timolol.

Hasta que no se disponga de más evidencias sobre la aparición de hiperemia con el uso de las combinaciones fijas de bimatoprost/timolol y travoprost/timolol, los resultados de este metaanálisis sugieren que a día de hoy la mejor alternativa a emplear cuando se necesita utilizar una combinación de análogos de prostaglandinas y timolol, es la combinación de latanoprost/timolol.

Una de las limitaciones de esta estimación es que se han incluido únicamente 8 ensayos clínicos y que el grupo comparador podía variar en cada uno de ellos, que podría incrementar la heterogeneidad de los distintos estudios incluidos. Debido a este grado de heterogeneidad encontrado entre los estudios incluidos, las conclusiones de este meta-análisis hay que tomarlas con cierta prudencia hasta que no se disponga de más ensayos clínicos comparativos que evalúen la aparición de hiperemia cuando se utilicen las distintas asociaciones fijas de protaglandinas y timolol.

Otra de las limitaciones de este estudio (y de cualquier revisión sistemática) es la posibilidad de estar excluyendo algún estudio publicado respecto al tema a tratar. La realización de una búsqueda exhaustiva en distintas bases de datos (Medline, Embase y Cochrane Controlled Clinical Trials Register) y la no utilización de sesgos de restricción (por tipo de publicación o idioma) ha tratado de minimizar este efecto. El período de tiempo empleado en la búsqueda (2000-2007) cubre perfectamente cualquier posible publicación, dadas las fechas de lanzamiento al mercado de los fármacos antiglaucomatosos en combinación fija.

Respecto a la existencia de un posible sesgo de publicación, es decir que los estudios con resultados negativos no se hubiesen publicado, el gráfico 
de embudo no señaló que esto estuviese ocurriendo, si bien su utilidad es limitada cuando el número de estudios incluidos es pequeño.

Los resultados obtenidos en este estudio se han centrado en analizar el grado de hiperemia que presenta latanoprost/timolol respecto a un conjunto de posibles fármacos en combinación (fija o no fija). $\mathrm{Si}$ en un futuro se dispusiese de un conjunto de ensayos clínicos frente a un mismo grupo comparador, sería interesante volver a realizar este metaanálisis, centrando el análisis en las comparaciones «face to face», que por el momento no es posible realizar.

Como conclusión, este metaanálisis ha mostrado que la combinación fija de latanoprost/timolol presenta una ventaja con respecto a otras alternativas existentes, al reducir significativamente el grado de hipermia que pueden padecer los pacientes como efecto secundario de los tratamientos. Este evento adverso debería ser tenido en cuenta, junto con los datos de eficacia, con el fin de mejorar el cumplimiento y la calidad de vida en un tipo de pacientes que debe estar en tratamiento de por vida, dada la cronicidad del glaucoma.

\section{BIBLIOGRAFÍA}

1. El éxito en la lucha contra las enfermedades infecciosas y el envejecimiento de la población modifican el perfil epidemiológico mundial de la ceguera. [citado 22 Mayo 2008]. Disponible en: www.who.int/mediacentre/news/ notes/2004/np27/es/

2. Kroese M, Burton H, Vardy S, Rimmer T, McCarter D. Prevalence of primary open angle glaucoma in general ophthalmic practice in the United Kingdom. Br J Ophthalmol 2002; 86: 978-980.

3. Antón A, Andrada MT, Mujica V, Calle MA, Portela J, Mayo A. Prevalence of primary open-angle glaucoma in a Spanish population: the Segovia study. J Glaucoma 2004; 13: 371-376.

4. Friedman DS, Jampel HD, Muñoz B, West SK. The prevalence of open-angle glaucoma among blacks and whites 73 years and older: the Salisbury Eye Evaluation Glaucoma Study. Arch Ophthalmol 2006; 124: 1625-1630.

5. Mayo Foundation for Medical Education and Research. Glaucoma [sede Web. [actualizado ]. Jul 17, 2008, acceso 20 de Marzo de 2009]. Disponible en: http://www.mayoclinic.com/health/glaucoma/ds00283/dsection =risk-factors

6. Kass MA, Heuer DK, Higginbotham EJ, Johnson CA, Keltner JL, Miller JP, et al. The Ocular Hypertension Treatment Study: a randomized trial determines that topical ocular hypotensive medication delays or prevents the onset of primary open-angle glaucoma. Arch Ophthalmol 2002: 120: 701-713.
7. European Glaucoma Society. Terminología y pautas en el glaucoma. 2. ${ }^{a}$ ed. Savona (Italia): Dogma, 2003.

8. American Academy of Ophtalmology. Primary OpenAngle Glaucoma, Preferred Practice Pattern. San Francisco: American Academy of Ophtalmology 2005. [citado 22 Mayo 2008]. Disponible en: www.aao.org/ppp

9. Friedman DS, Quigley HA, Gelb L, Tan J, Margolis J, Shah SN, et al. Using pharmacy claims data to study adherence to glaucoma medications: methodology and findings of the Glaucoma Adherence and Persistency Study (GAPS). Invest Ophthalmol Vis Sci 2007; 48: 5052-5057.

10. Day DG, Sharpe ED, Atkinson MJ, Stewart JA, Stewart $W C$. The clinical validity of the treatment satisfaction survey for intraocular pressure in ocular hypertensive and glaucoma patients. Eye 2006; 20: 583-590.

11. Joel S. Schuman, MD. Antiglaucoma medication: a review of safety and tolerability issues related to their use. Clin Ther 2000; 22: 167-208.

12. Li N, Chen XM, Zhou Y, Wei ML, Yao X. Travoprost compared with other prostaglandin analogues or timolol in patients with open-angle glaucoma or ocular hypertension: meta-analysis of randomized controlled trials. Clin Exp Ophthalmol 2006; 34: 755-764.

13. Feldman RM. Conjunctival hyperemia and the use of topical prostaglandins in glaucoma and ocular hypertension. J Ocul Pharmacol Ther 2003; 19: 23-35.

14. Jadad AR, Moore RA, Carroll D, Jenkinson C, Reynolds $D J$, Gavaghan DJ, et al. Assessing the quality of reports of randomized clinical trials: is blinding necessary? Control Clin Trials 1996; 17: 1-12.

15. Higgins JP, Thompson SG, Deeks JJ, Altman DG. Measuring inconsistency in meta-analyses. BMJ 2003; 327: 557-560.

16. Mantel J, Haenszel W. Statistical aspects of the analysis of data from retrospective studies of disease. $J$ Natl Cancer Inst 1959; 22: 719-748.

17. Der Simonian R, Laird N. Meta-analysis in clinical trials. Control Clin Trials 1986; 7: 177-188.

18. Martín JL, Martín-Sánchez E, Torralba E, Díaz E, Lurueña-Segovia S, Alonso EJ. Investigación secundaria: revisión sistemática y metaanálisis. Semergen 2008; 34: 11-16.

19. Moher D, Cook DJ, Eastwood S, Olkin I, Rennie D, Stroup $D F$, et al. Improving the quality of reports of meta-analyses of randomised controlled trials: the QUORUM statement. Lancet 1999; 354: 1896-1900.

20. Delgado M, Llorca D, Doménech JM. Estudios de cohortes. Metodología de la investigación en ciencias de la salud. $3^{a}$ ed. Barcelona: Signo; 2008.

21. Konstas AG, Bányai L, Blask KD, Väth J, Kozobolis VP, Trüb PR, et al. Intraocular pressure and safety in glaucoma patients switching to latanoprost/timolol maleate fixed combination from mono- and adjunctive therapies. J Ocul Pharmacol Ther 2004; 20:375-382.

22. Hamacher T, Schinzel M, Schölzel-Klatt A, Neff HM, Maier H, Schlaffer G, et al. Short term efficacy and safety in glaucoma patients changed to the latanoprost $0.005 \% /$ timolol maleate $0.5 \%$ fixed combination from monotherapies and adjunctive therapies. Br J Ophthalmol 2004; 88: 1295-1298.

23. Konstas AG, Nakos E, Tersis I, Lallos NA, Leech JN, Stewart WC. A comparison of once-daily morning vs evening dosing of concomitant latanoprost/timolol. Am J Ophthalmol 2002; 133: 753-757. 
24. Cvenkel B, Stewart JA, Nelson LA, Stewart WC. Dorzolamide/timolol fixed combination versus latanoprost/timolol fixed combination in patients with primary open-angle glaucoma or ocular hypertension. Curr Eye Res 2008; 33: 163-168.

25. Stewart WC, Stewart JA, Day DG, Sharpe ED, Jenkins JN. Efficacy and safety of the latanoprost/timolol maleate fixed combination vs concomitant brimonidine and latanoprost therapy. Eye 2004; 18: 990-995.

26. Stewart WC, Stewart JA, Day D, Sharpe ED. Efficacy and safety of timolol maleate/latanoprost fixed combination versus timolol maleate and brimonidine given twice daily. Acta Ophthalmol Scand 2003; 81:242-246.

27. Konstas AG, Kozobolis VP, Lallos N, Christodoulakis E, Stewart JA, Stewart WC. Daytime diurnal curve comparison between the fixed combinations of latanoprost $0.005 \% /$ timolol maleate $0.5 \%$ and dorzolamide $2 \% /$ timolol maleate 0.5\%. Eye 2004; 18: 1264-1269.

28. Konstas AG, Boboridis K, Tzetzi D, Kallinderis K, Jenkins $J N$, Stewart WC. Twenty-four-hour control with latanoprost-timolol-fixed combination therapy vs latanoprost therapy. Arch Ophthalmol 2005; 123: 898-902.

29. Shin DH, Feldman RM, Sheu WP; Fixed Combination Latanoprost/Timolol Study Group. Efficacy and safety of the fixed combinations latanoprost/timolol versus dorzolamide/timolol in patients with elevated intraocular pressure. Ophthalmology 2004; 111: 276-282.

30. Diestelhorst M, Larsson LI; European Latanoprost Fixed Combination Study Group. A 12 week study comparing the fixed combination of latanoprost and timolol with the concomitant use of the individual components in patients with open angle glaucoma and ocular hypertension. $\mathrm{Br} J \mathrm{Oph}$ thalmol 2004; 88: 199-203.

31. Martinez A, Sanchez M. A comparison of the safety and intraocular pressure lowering of bimatoprost/timolol fixed combination versus latanoprost/timolol fixed combination in patients with open-angle glaucoma. Curr Med Res Opin 2007; 23: 1025-1032.

32. Pfeiffer N. A comparison of the fixed combination of latanoprost and timolol with its individual components. Graefe's Arch Clin Exp Ophthalmol 2002; 240: 893-899.

33. Higginbotham EJ, Feldman R, Stiles M, Dubiner H; Fixed Combination Investigative Group. Latanoprost and timolol combination therapy vs monotherapy. Arch Ophthalmol 2002; 120: 915-922.
34. Olander K, Zimmerman TJ, Downes N, Schoenfelder J; Xalacom/Latanoprost Study Group. Switching from latanoprost to fixed-combination latanoprost-timolol: A 21day, randomized, double-masked, active-control study in patients with glaucoma and ocular hypertension. Clin Ther 2004; 26: 1619-1629.

35. Franks WA, Renard JP, Cunliffe IA, Rojanapongpun P. A 6-week, double-masked, parallel-group study of the efficacy and safety of travoprost $0.004 \%$ compared with latanoprost $0.005 \%$ /timolol $0.5 \%$ in patients with primary open-angle glaucoma or ocular hypertension. Clin Ther 2006; 28: 332-339.

36. García-Sánchez, J, Rouland JF, Spiegel D, Pajic B, Cunliffe I, Traverso C, et al. A comparison of the fixed combination of latanoprost and timolol with the unfixed combination of brimonidine and timolol in patients with elevated intraocular pressure. A six month, evaluator masked, multicentre study in Europe. Br J Ophthalmol 2004; 88: 877883.

37. Diestelhorst M, Larsson LI; European-Canadian Latanoprost Fixed Combination Study Group. A 12-week, randomized, double-masked, multicenter study of the fixed combination of latanoprost and timolol in the evening versus the individual components. Ophthalmology 2006; 113: 7076.

38. Martinez-de-la-Casa JM, Castillo A, Garcia-Feijoo J, Mendez-Hernandez, C, Fernandez-Vidal A, Garcia-Sanchez J. Concomitant administration of travoprost and brinzolamide versus fixed latanoprost/timolol combined therapy: three-month comparison of efficacy and safety. Curr Med Res Opin 2004; 20: 1333-1339.

39. Topouzis F, Melamed S, Danesh-Meyer H, Wells AP, Kozobolis V, Wieland $H$, et al. A 1-year study to compare the efficacy and safety of once-daily travoprost $0.004 \% /$ timolol $0.5 \%$ to once-daily latanoprost $0.005 \% /$ timolol $0.5 \%$ in patients with open-angle glaucoma or ocular hypertension. Eur J Ophthalmol 2007; 17: 183-190.

40. Popoviç Suiç S, Cerovski B, Jukiç T. The role of patient compliance in the management of glaucoma. Acta Med Croatica 2008; 62: 57-59.

41. Feldman RM. Conjunctival hyperemia and the use of topical prostaglandins in glaucoma and ocular hypertension. J Ocul Pharmacol Ther 2003; 19: 23-35.

42. Hollo $G$. The side effects of the prostaglandin analogues. Expert Opin Drug Saf 2007; 6: 45-52. 
